\title{
La autonomía económica de las mujeres latinoamericanas
}

\author{
Edith Johana Medina Hernández* \\ María José Fernández Gómez**
}

Fecha de recepción: 21 de marzo de 2021

Fecha de aprobación: 21 de junio de 2021

Resumen: El acceso al trabajo remunerado, la ocupación según sectores de productividad y el uso del tiempo son factores que influyen en la capacidad de las mujeres para generar ingresos propios, por lo cual son condicionantes de la autonomía económica y deberían presentarse en igualdad de condiciones frente a los hombres. El principal objetivo de esta investigación es conocer el posicionamiento laboral de las mujeres latinoamericanas a través del análisis de seis indicadores de ocupación en 15 países de la región, los cuales fueron publicados en el año 2020 por el Observatorio de Género de la Comisión Económica para América Latina y el Caribe (CEPAL). Mediante el uso de la técnica multivariante HJ-Biplot se exploran similitudes y diferencias entre indicadores, países y géneros, para conocer las condiciones que no favorecen el empoderamiento económico de la mujer ni su participación en el mercado laboral. Se concluye que las naciones centroamericanas registran las mayores brechas de autonomía económica tanto entre los hombres como entre las mujeres de Latinoamérica, mientras que en países como Uruguay, Colombia, Costa Rica, Chile, Panamá y Brasil se registran mayores oportunidades de ocupación laboral femenina en sectores de alta productividad.

Palabras clave: sociología económica, mujer y desarrollo, división sexual del trabajo, indicadores de desarrollo, América Latina, análisis multivariado.

Clasificación JEL: J16, J22, R58, C39, Y10

Cómo citar

Medina Hernández, E. J., \& Fernández Gómez, M. J. (2021). La autonomía económica de las mujeres latinoamericanas. Apuntes del Cenes, 40(72). Págs. 181 - 204. https://doi.org/10.19053/01203053.v40.n72.2021.12606

* Ph.D. (C) Estadística Multivariante Aplicada de la Universidad de Salamanca. Máster en Análisis Avanzado de Datos Multivariantes. España. Contacto: edith.medina@usal.es. iD https://orcid.org/0000-0003-4207-0333.

** PhD. Estadística. Profesora titular del Departamento de Estadística, Universidad de Salamanca. España. mjfg@usal.es iDhttp://orcid. org/0000-0002-5530-6416. 


\title{
Economic Autonomy of Latin American Women
}

\begin{abstract}
Access to paid work, the occupation according to productivity sectors and the time use are factors that influence the ability of women to generate their own income, therefore, they conditionate the economic autonomy and should be presented on equal terms with respect to men. The main objective of this research is to know the labor position of Latin American women through the analysis of 6 employment indicators in 15 countries of the region, which were compiled and published in 2020 by the Gender Observatory of the Economic Commission for Latin America and the Caribbean (ECLAC). Through the HJ-Biplot multivariate technique, similarities and differences between indicators, countries and genders were explored, to find out the conditions that do not favor the economic empowerment of women or their participation in the labor market. It is concluded that the Central American nations register the largest gaps in economic autonomy both between men and women in Latin America, while in countries such as Uruguay, Colombia, Costa Rica, Chile, Panama and Brazil, there are increased opportunities for female employment in high productivity sectors.
\end{abstract}

Keywords: economic sociology, women and development, sexual division of labour, development indicators, Latin America, multivariate analysis. 


\section{INTRODUCCIÓN}

En el 2015, 193 países del mundo adoptaron la Agenda 2030 para el Desarrollo Sostenible y establecieron 17 Objetivos (ODS), entre los cuales se registra de forma transversal el enfoque de género como una condición necesaria para el progreso de las naciones.

Las metas sobre la igualdad de género están integradas en 11 de los 17 objetivos (en diferentes niveles) y uno de ellos aborda explícitamente la igualdad de género -ODS 5: Lograr la igualdad entre los géneros y empoderar a todas las mujeres y las niñas-. (Morgan et al., 2020, p. 205)

También los objetivos 8 y 10 se relacionan con cuestiones de género y enfatizan la necesidad de que las mujeres accedan a un trabajo decente, a fin de reducir las desigualdades que puedan limitar su empoderamiento y liderazgo socioeconómico.

Según especifican Altuzarra et al. (2021), "Los Objetivos de Desarrollo Sostenible de las Naciones Unidas para 2015-2030 establecen estándares globales para lograr la igualdad de género y alentar a los gobiernos y la sociedad civil a implementar políticas destinadas a alcanzar estos estándares" (p. 382). Estos estándares buscan reconocer que las condiciones de igualdad de género contribuyen al desarrollo de las naciones al impulsar las economías sostenibles.

Específicamente, en el marco del desarrollo de los ODS 5, 8 y 10, se analiza la autonomía económica de las mujeres, entendida como "la capacidad para generar ingresos y finanzas personales desde recursos provenientes del trabajo remunerado" (CEPAL, 2017, p.15). La autonomía económica tiene en cuenta el uso del tiempo, la contribución de la economía del cuidado al desarrollo sostenible y la participación de la mujer en los sectores productivos, de forma que "supone avanzar en la posibilidad de definir y participar en la toma de las decisiones" (Micha, 2019, p. 365).

Por ello, el estudio de los indicadores asociados a la dimensión económica de género ha suscitado un gran interés en el ámbito sociopolítico de los 
últimos años, pues permite dimensionar el empoderamiento de la mujer en la sociedad.

En el discurso político internacional, se destaca la relevancia de la autonomía económica para el logro del empoderamiento de las mujeres. Pero a la vez se plantea la diversidad de contextos sociales, económicos y culturales donde habitan las mujeres, así como las desiguales condiciones en que se enfrentan al trabajo remunerado. (Calcagni \& Cortínez, 2016, p. 5)

El desarrollo de la autonomía económica de las mujeres debería estar determinado por la igualdad de condiciones frente a los hombres, pero existen circunstancias de índole cultural que no favorecen la igualdad, por lo que se hace necesario medir el nivel de paridad existente, así como la evolución de su empoderamiento. En el contexto latinoamericano, la Comisión Económica para América Latina y el Caribe (CEPAL) es una de las instituciones internacionales que estudia el desarrollo de género, haciendo un seguimiento de las estadísticas oficiales de los países de la región. Mediante las cifras que compila y publica regularmente esta institución, es posible estudiar por ejemplo que "cuando las mujeres acceden al mercado laboral en igualdad de oportunidades y de trato con los hombres, tienen la posibilidad real de desarrollar todas sus potencialidades, reforzar su autoestima, definir su identidad y ejercer plenamente la ciudadanía" (Goyes, 2019, p. 198).

Dada la pertinencia de estudiar la autonomía económica femenina, en este trabajo se examinan seis indicadores de ocupación, con el objetivo de analizar el posicionamiento laboral de las mujeres latinoamericanas, mediante la técnica de análisis multivariante HJ-Biplot, las similitudes y diferencias observadas entre indicadores, géneros, países y las características que las determinan. En este sentido, este estudio contribuye al estado del tema de la investigación de género en la región, en la medida en que presenta un análisis multidimensional de los últimos datos sobre autonomía económica de las mujeres. El estudio de dichos índices, tanto en América Latina como en el Caribe, suele hacerse por separado, de forma descriptiva o bivariante, mas no de forma conjunta, como se aborda en este artículo1.

En este trabajo se busca responder estos tres interrogantes: ¿en qué indicadores de autonomía económica se registran las mayores diferencias entre géneros?, ¿en cuáles de ellos no se perciben diferencias estadísticamente significativas?, y ¿en qué países de América Latina y del Caribe se observan mayores oportunidades para el empoderamiento económico de la mujer?

1 En el sistema de estadísticas CEPALSTAT, disponible en: https://oig.cepal.org/es/indicadores, es posible acceder a las cifras de cada indicador. Allí se presentan gráficos descriptivos de cada uno de estos sin interpretar relaciones de interdependencia entre ellos. 
Para dar respuesta a estas preguntas, este documento se organiza según la siguiente estructura. En primer lugar, se presenta una revisión del estado del tema de los estudios de la autonomía económica en el contexto latinoamericano, en los que se han realizado análisis de indicadores de género. Posteriormente, se presenta el método de estudio, donde se incluyen tanto las características generales de los datos analizados como una breve explicación de la técnica de análisis utilizada, el HJ-Biplot. A continuación, se describen los resultados de la investigación, comenzando por una exploración descriptiva de los indicadores de autonomía económica y las correlaciones bivariadas que se observan entre ellos, y presentando después los resultados del análisis HJ-Biplot. Al final se expone la discusión sobre las implicaciones de los hallazgos y las conclusiones obtenidas.

\section{REVISIÓN DE LITERATURA}

Históricamente, la mayor desigualdad entre hombres y mujeres en las distintas esferas en las que pueden desarrollar su autonomía se ha registrado en el acceso al mercado de trabajo (formal o informal), que demarca condiciones salariales no óptimas para algunas mujeres. Tal como explica Vaca Trigo (2019),

la menor participación de las mujeres en el empleo, su concentración en sectores de menor productividad y el acceso a empleos informales o de menor calidad, son factores que tienen un impacto significativo en su posibilidad de generar ingresos propios, limitando su autonomía económica. (p. 29)

Incluso en aspectos específicos de la autonomía femenina, como puede ser el uso del tiempo, se registran desigualdades, pues las mujeres suelen tener menos tiempo disponible para acceder a medios que les permitan generar recursos, como consecuencia de actividades de cuidado del hogar. Bidegain (2017) apunta que "La autonomía económica que se vincula con la posibilidad de controlar los activos y recursos y liberar a las mujeres de la responsabilidad exclusiva de las tareas reproductivas y de cuidado" (p. 24), lo cual es clave para el empoderamiento económico de la mujer.

Hagqvist et al. (2019) indican que "si las mujeres eligen el trabajo por cuenta propia como un medio para equilibrar el trabajo y la familia, deberían dedicar menos horas al trabajo remunerado y más horas al trabajo no remunerado" (p. 684). Al respecto, Meyer (2019) añade:

La incorporación de las mujeres al mercado laboral no ha ido acompañada del ingreso de los hombres al espacio doméstico y de cuidado. Por lo que se ha generado una acumulación de roles y responsabilidades que recae principalmente en las mujeres. En este marco, surge la metáfora de la "mujer pulpo", la cual refiere a la capacidad "natural" de las mujeres de ser madres, trabajadoras, esposas, parejas, cuida- 
doras, dirigentes [sic], todo al mismo tiempo. (p. 86)

Estos autores aluden a la importancia de analizar el acceso que tienen las mujeres al mercado del trabajo y cómo este último se puede ver afectado por la economía del cuidado en los hogares, la cual también les resta tiempo para el desarrollo de sus aspiraciones personales. De Clercq y Brieger (2021) argumentan al respecto que un incremento en la autonomía económica y laboral de las mujeres aumenta la probabilidad de que se sientan satisfechas con su capacidad para equilibrar las necesidades de trabajar con los aspectos de su vida personal. Sin embargo, esta condición en países como los latinoamericanos resulta inequitativa.

Por ello, y con la intención de delimitar el estado del tema de este estudio, es pertinente hacer referencia a algunos de los autores que analizan la dimensión económica del género, la incorporación de las mujeres al mercado del trabajo y el comportamiento de la autonomía económica femenina desde la interpretación de indicadores, en el contexto de América Latina. Entre ellos, Varea (2020), Sehnbruch et al. (2020), Htun et al. (2019), Liu et al. (2017), Lamelas y Aguay (2010) y DANE (2020).

Varea (2020), al estudiar la problemática de género con relación a los objetivos de desarrollo sostenible, afirma que más del $50 \%$ de las mujeres de América Latina percibe ingresos inferiores a un salario mínimo, lo cual, en el contexto del ODS 8, implica que "se deben analizar cuáles son las razones que dan las mujeres para no estar en el mercado laboral, es decir, las responsabilidades familiares" (p. 123), ya que es importante entender de qué forma las brechas salariales afectan a las sociedades latinoamericanas.

Sehnbruch et al. (2020) estudian la calidad del empleo en nueve países de América Latina, considerando la informalidad del empleo en la región y según un enfoque de capacidades. Utilizan el método de Alkire y Foster (AF), para construir un indicador sintético de la calidad del empleo (que denominan QoE) a nivel individual, el cual resume tres dimensiones: ingresos, seguridad laboral y condiciones laborales. Estos autores observan que hay heterogeneidad en las condiciones laborales entre países, porque, por ejemplo, Chile, Perú, Colombia y Brasil tienen altas tasas de rotación laboral. En relación con el género, su estudio concluye que existen brechas entre hombres y mujeres en cuanto a la calidad laboral, situación que se vuelve compleja al comparar entre países, porque exceptuando a México y Uruguay, las mujeres tienen privaciones más intensas en términos de su QoE.

Htun et al. (2019) estudian cómo, a pesar de que en los últimos años se han producido avances generalizados en los derechos de la mujer, en muchos países aún siguen arraigadas restricciones a 
su autonomía, por lo que es apropiado explorar la asociación existente entre la legislación discriminatoria de género y los indicadores de autonomía económica femenina. Estos autores concluyen que las restricciones de índole legal predicen la propiedad de activos de las mujeres y su participación en la fuerza laboral, mientras que la discriminación en el trabajo asalariado está asociada con el tamaño y la dirección de las diferencias salariales. Estos hallazgos resaltan la importancia de conceptualizar, describir y analizar variables relacionadas con el ámbito económico de la mujer.

Liu et al. (2017) analizan cómo ha aumentado desde 1970 el liderazgo femenino representado en mujeres cabeza de familia en los hogares latinoamericanos. Utilizan microdatos censales de la Serie Microdata-International IPUMS-I ${ }^{2}$ para comparar el comportamiento de 14 países y examinan factores condicionantes de pobreza de las mujeres entre $35 \mathrm{y}$ 44 años, presencia de hijos en el hogar, tipo de residencia (urbana o rural), propiedad de la vivienda, sexo del jefe del hogar, estado civil, edad y logros educativos. En este estudio se concluye que las mujeres tienen cada vez más probabilidades de ser cabeza de familia, al residir en hogares considerados como no pobres, una vez controladas las condiciones maritales; es decir, por ejemplo, la monoparentalidad, el divorcio o la convivencia no matrimonial.
Como referencia histórica se cita a Lamelas y Aguayo (2010), quienes midieron la desigualdad de género en Latinoamérica desde la perspectiva de los indicadores de desarrollo humano desde 1997 a 2007. Dicho trabajo presentó una panorámica comparativa de la evolución entre los países latinoamericanos, que cuestionaba la relación entre el índice de desarrollo de género (IDG) y el índice de potenciación de género (IPG). Mediante análisis estadísticos descriptivos y pruebas de hipótesis, se encontraron diferencias significativas entre los ingresos estimados por género en los hogares, que fueron cercanos al $17 \%$ para el caso de las mujeres y al $50 \%$ para los hombres.

Ahora bien, en el contexto específico de Colombia es posible referenciar el informe DANE (2020), donde se compara la tasa de participación económica de las mujeres colombianas durante el periodo 2008-2019 y se indica que se incrementó alrededor de siete puntos porcentuales, pues pasó del $46.4 \%$ al $53.1 \%$. Otras cifras de interés en este estudio (2020) son las referidas a la posición 22 que ocupa Colombia con respecto a 153 países en el índice global de brechas de género; además, se resalta que "las mujeres reciben por su trabajo ingresos $12.1 \%$ menores que los hombres" (p. 23).

2 Compilada por el Centro de Población de Minnesota, Estados Unidos. 


\section{MATERIALES Y MÉTODOS}

Este estudio es exploratorio, descriptivo e inferencial, ya que se busca encontrar relaciones y diferencias por géneros en el comportamiento de los distintos indicadores de autonomía económica que se compilan en el Observatorio de Género de la CEPAL, los cuales se resumen en la Tabla 1. En el análisis se utilizan los datos correspondientes al año 2020 para 15 países, ${ }^{3}$ con la intención de identificar en cuáles de ellos se percibe un mayor fomento de la igualdad de oportunidades económicas entre los y las latinoamericanas. Estos son Argentina, Brasil, Chile, Colombia, Costa Rica, Ecuador, El Salvador, Guatemala, Honduras, México, Panamá, Paraguay, Perú, República Dominicana y Uruguay.

Tabla 1. Indicadores en análisis

\begin{tabular}{|c|c|c|c|}
\hline Indicador & Forma de cálculo & Observación & Escala \\
\hline $\begin{array}{l}\text { PSIP. Porcentaje de } \\
\text { población sin ingresos } \\
\text { propios }\end{array}$ & $\begin{array}{l}\text { Proporción de la población femenina o } \\
\text { masculina de } 15 \text { años y más que no es } \\
\text { perceptora de ingresos monetarios, en relación } \\
\text { con el total de la población de hombres o } \\
\text { mujeres de } 15 \text { años y más. El resultado se } \\
\text { expresa en porcentajes. }\end{array}$ & $\begin{array}{l}\text { Estimado sobre la base de } \\
\text { encuestas de hogares de los } \\
\text { países, pertenecientes al Banco } \\
\text { de Datos de Encuestas de } \\
\text { Hogares (BADEHOG). }\end{array}$ & $\begin{array}{c}5.8 \mathrm{a} \\
51\end{array}$ \\
\hline $\begin{array}{l}\text { TTR. Tiempo promedio } \\
\text { destinado al trabajo } \\
\text { remunerado para población } \\
\text { de } 15 \text { años o más }\end{array}$ & $\begin{array}{l}\text { El trabajo remunerado se refiere al tiempo } \\
\text { dedicado al empleo, a su búsqueda y al } \\
\text { traslado al trabajo. }\end{array}$ & $\begin{array}{l}\text { Horas semanales reportadas en } \\
\text { la última encuesta nacional de } \\
\text { uso del tiempo en cada país. }\end{array}$ & $\begin{array}{c}14.2 \mathrm{a} \\
44.9\end{array}$ \\
\hline $\begin{array}{l}\text { T T NR. Tiempo no } \\
\text { remunerado dedicado a los } \\
\text { quehaceres domésticos y } \\
\text { de cuidado del hogar }\end{array}$ & $\begin{array}{l}\text { El trabajo no remunerado se realiza sin pago } \\
\text { alguno. Cuantifica el tiempo que una persona } \\
\text { dedica a las labores domésticas y de cuidados } \\
\text { del hogar. }\end{array}$ & $\begin{array}{l}\text { Horas semanales reportadas en } \\
\text { la última encuesta nacional de } \\
\text { uso del tiempo en cada país. }\end{array}$ & $\begin{array}{l}5.7 \text { a } \\
53.9\end{array}$ \\
\hline $\begin{array}{l}\text { P OP B. Población } \\
\text { ocupada en sectores de } \\
\text { productividad baja }\end{array}$ & $\begin{array}{l}\text { Cociente entre la población ocupada de } 15 \\
\text { años y más que desempeña actividades } \\
\text { laborales en agricultura, comercio o servicios, } \\
\text { y el total de la población ocupada de } 15 \text { años y } \\
\text { más. El resultado se multiplica por } 100 \text {. }\end{array}$ & & $\begin{array}{c}49.3 \mathrm{a} \\
84.7\end{array}$ \\
\hline $\begin{array}{l}\text { P OPM. Población } \\
\text { ocupada en sectores de } \\
\text { productividad media }\end{array}$ & $\begin{array}{l}\text { Cociente entre la población ocupada de } 15 \\
\text { años que labora en industria manufacturera, } \\
\text { construcción, transporte o comunicaciones, y } \\
\text { el total de la población ocupada de } 15 \text { años y } \\
\text { más. El resultado se multiplica por } 100 \text {. }\end{array}$ & $\begin{array}{l}\text { Los sectores de actividad } \\
\text { económica se han determinado } \\
\text { de acuerdo con la Clasificación } \\
\text { Industrial Internacional Uniforme } \\
\text { de todas las Actividades } \\
\text { Económicas (CIIU, Rev.2). }\end{array}$ & $\begin{array}{l}10 a \\
40.6\end{array}$ \\
\hline $\begin{array}{l}\text { POPA. Población } \\
\text { ocupada en sectores } \\
\text { de productividad alta }\end{array}$ & $\begin{array}{l}\text { Cociente entre la población ocupada de } 15 \text { años } \\
\text { y más que trabaja en minería, electricidad, gas } \\
\text { y agua, actividades financieras e inmobiliarias, } \\
\text { y el total de la población ocupada de } 15 \text { años y } \\
\text { más El resultado se multiplica por } 100 \text {. }\end{array}$ & & $\begin{array}{c}3.5 \mathrm{a} \\
14.1\end{array}$ \\
\hline
\end{tabular}

Fuente: elaboración propia con base en información Cepal (2020).

3 Las fuentes de información desde las cuales reúne las cifras de cada país el Observatorio Regional de Género, varían entre sí, al igual que los años de medición de cada indicador oscilan entre 2017 y 2020 . Esto porque en Latinoamérica no existe estandarización frente a la forma en que deberían reportar las estadísticas oficiales los diferentes gobiernos o instituciones encargadas de su producción. En este sentido, las variables seleccionadas en este análisis pueden ser susceptibles de valores atípicos asociados a diferentes años de medición y distintos estados de ciclo económico de los países. 
Para presentar los resultados, se interpreta el comportamiento de los indicadores de forma individual y mediante su análisis multidimensional a través de la técnica HJ-Biplot, en la que se considera la estructura de los datos examinados tal como se presenta en la Figura 1. Para entender el alcance de esta técnica es preciso discutir brevemente sus fundamentos y la forma en que se interpretan sus resultados, tanto numéricos como gráficos, por ello, a continuación se especifican los referentes metodológicos de este método de análisis multivariante.

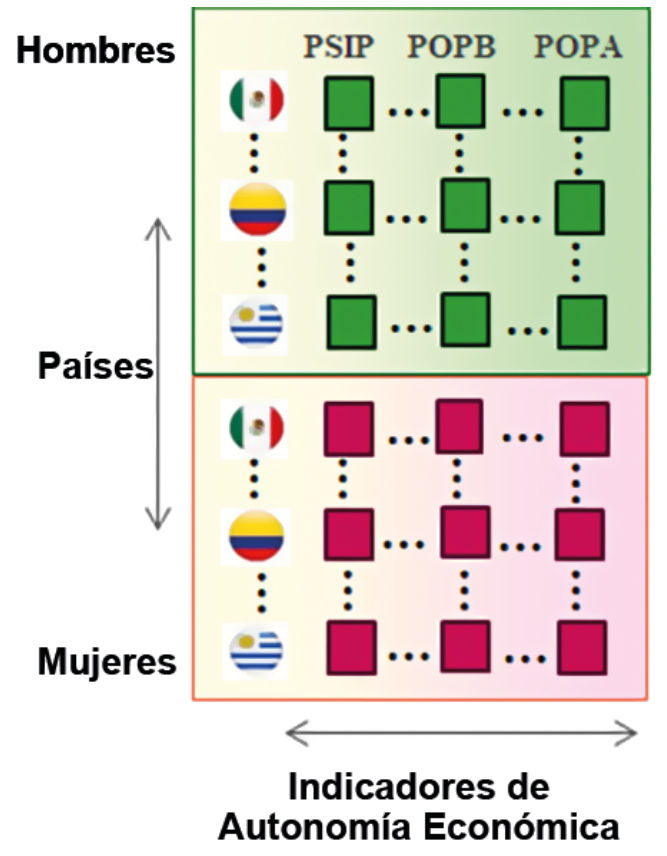

Figura 1. Estructura de la información en análisis.

Fuente: elaboración propia.

\section{La técnica HJ-Biplot}

Los métodos Biplot propuestos por Gabriel (1971), son un conjunto de técnicas de análisis multivariante que permiten aproximar una matriz de datos por otra de baja dimensión, donde es posible representar mediante marca- dores a los individuos (observaciones o filas en la tabla de datos) y las variables (columnas). Este autor propone dos métodos, el GH-Biplot y el JK-Biplot. El primero consigue una alta calidad en la representación de las variables (que regularmente se grafican mediante vectores), mientras que el JK-Biplot logra 
una alta calidad en la representación de los individuos (los cuales se representan habitualmente como puntos).

Por su parte, Galindo (1986) propone el HJ-Biplot como alternativa para representar simultáneamente las filas (que en este estudio corresponden a los países latinoamericanos en análisis) y las columnas (indicadores de género analizados) de la matriz, sobre un mismo sistema de ejes cartesianos, con lo cual consigue la máxima calidad de representación tanto para los individuos (países) como para las variables (indicadores). En la Figura 2 se exponen los elementos presentes en este tipo de representaciones, en un espacio de dimensión reducida (generalmente bidimensional). Mediante dichos elementos se analizan los resultados en este estudio (los individuos se representan mediante puntos y las variables mediante vectores).

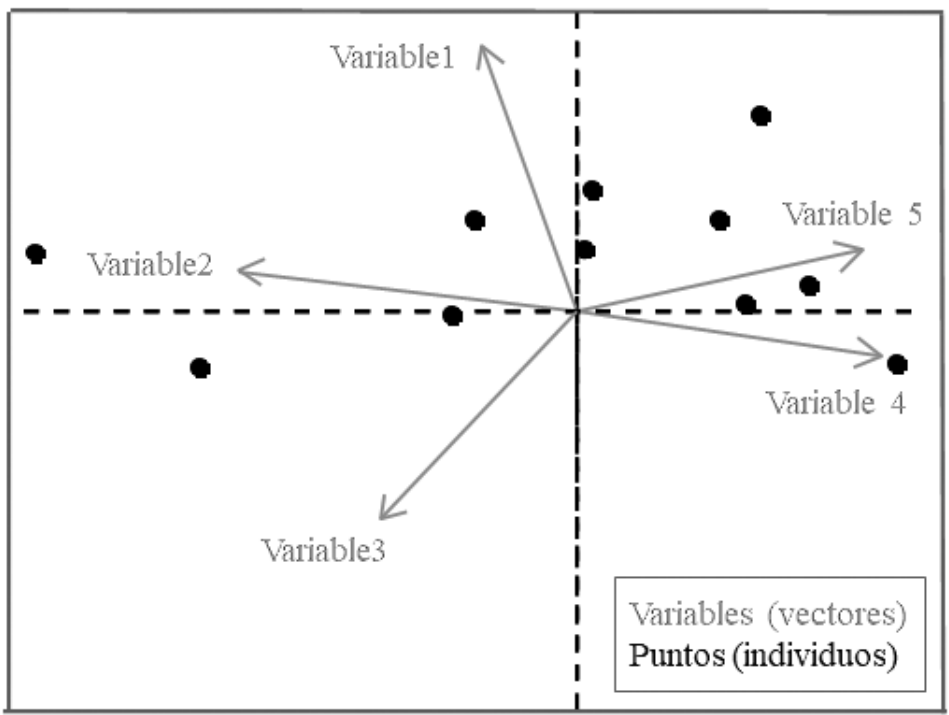

Figura 2. Elementos en las representaciones HJ-Biplot.

Fuente: elaboración propia con base en Díaz-Faes et al. (2013).

Para interpretar los resultados gráficos de un análisis Biplot, se analizan los siguientes elementos:

Los ángulos que forman los vectores que representan a las variables con los ejes indican cuáles son los elementos más característicos de dichos ejes. El ángulo entre vectores presenta la correlación entre las variables, es decir, si los vectores forman un ángulo agudo próximos entre sí, están altamente correlacionados de forma positiva, si son perpendiculares, entonces son inde- 
pendientes, y si se ubican en direcciones opuestas, tienen una alta correlación negativa o inversa.

La longitud de cada vector se asocia a la dispersión que presenta la variable a la que representa, de forma que, a mayor longitud del vector, mayor variabilidad, y viceversa. La distancia entre los puntos fila refleja en qué medida los individuos son similares entre sí, de forma que, si se ubican cerca, están determinados por características similares que se pueden explicar en relación con las variables representadas en el plano. La cercanía entre un punto y un vector refleja que dicho individuo es significativo para la explicación de la variable $\mathrm{y}$, a su vez, que la variable tiene un valor alto para discriminar al individuo.

\section{RESULTADOS}

A continuación, se presentan los resultados del análisis univariante. Inicialmente, se discuten las diferencias observadas entre géneros, para cada uno de los indicadores y según los 15 países estudiados. Posteriormente, se detallan los resultados del análisis multivariante mediante la interpretación de las representaciones surgidas del aná- lisis HJ-Biplot, al considerar los datos de ambos géneros en un mismo plano y cuando se hace su distinción en planos individuales. Se trata de describir si se observan características comunes entre grupos de países e identificar en cuáles de ellos se registran las mayores oportunidades de igualdad de género en el ámbito de la autonomía económica.

En la Figura 3 se muestran los porcentajes de población de 15 años o más sin ingresos propios (PSIP). Al comparar el comportamiento de este indicador según el género, se observa cómo en todas las naciones latinoamericanas es siempre mayor el indicador de mujeres sin fuente de ingresos, respecto a los hombres en la misma condición. La menor diferencia se observa en Uruguay (5.9\% de los hombres sin ingresos propios versus $13 \%$ de las mujeres), mientras que la mayor diferencia se registra en Guatemala (14\% frente a $51 \%$ ). Los países centroamericanos analizados reflejan los indicadores más altos de dependencia de ingreso para las mujeres. Para el caso de Colombia, puede considerarse que este país tiene un comportamiento promedio frente al total de los países latinoamericanos. 


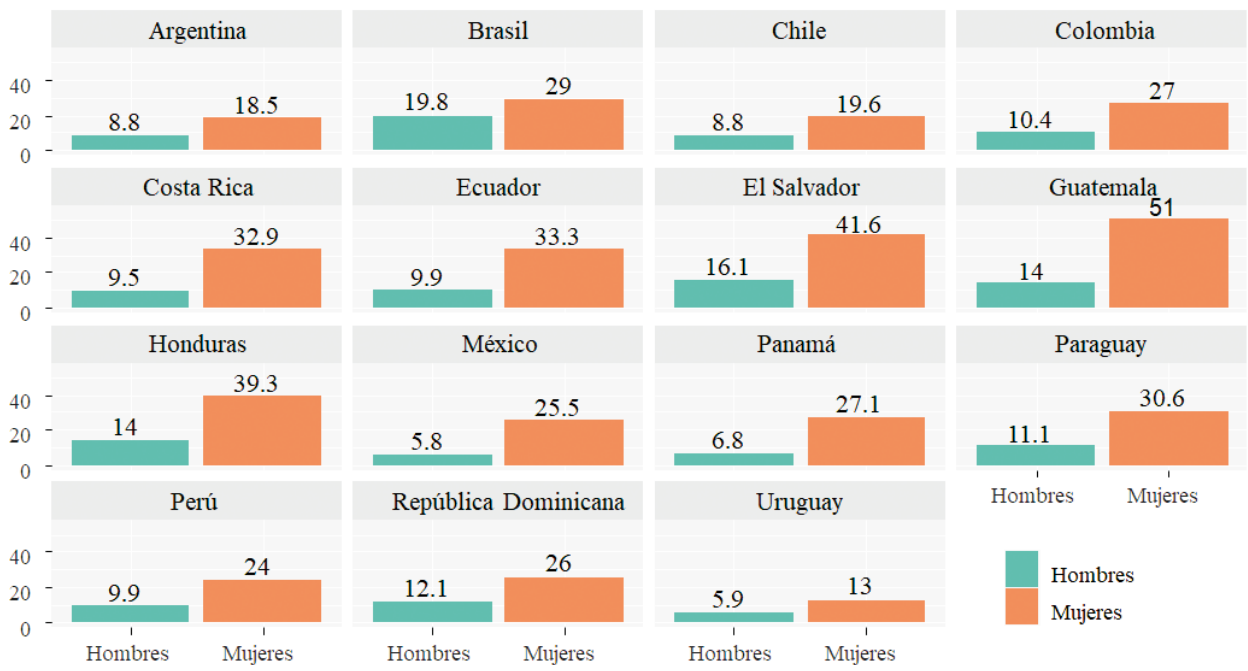

Figura 3. Población de 15 años y más sin ingresos propios por géneros.

Fuente: elaboración propia en software estadístico R con base en datos Cepal (2020).

En la Figura 4 se presentan los indicadores del tiempo promedio destinado al trabajo remunerado y no remunerado por países, para la población de 15 años y más, según género (indicadores TTR y TTNR). Para el caso de los tiempos de trabajo no remunerado, es decir, los que pueden atribuirse a las actividades de cuidado y mantenimiento del hogar, se observa que son siempre mayores las dedicaciones de tiempo de las mujeres. En ninguna nación los hombres declaran dedicar más de 20 horas semanales al trabajo no remunerado, mientras que tal dedicación llega a ser de hasta de 54 horas semanales entre las mujeres mexicanas.
En contraste, frente al tiempo de trabajo remunerado, las proporciones se invierten, de forma que siempre son mayores los tiempos promedio declarados por los hombres. La menor diferencia observada entre géneros para ambos tipos de tiempos de trabajo (remunerado y no), se registra en Brasil, donde las diferencias entre hombres y mujeres son cercanas a 11 horas semanales, mientras que la mayor desigualdad en el uso del tiempo se percibe en Guatemala, donde se registran brechas de aproximadamente 30 horas semanales. 

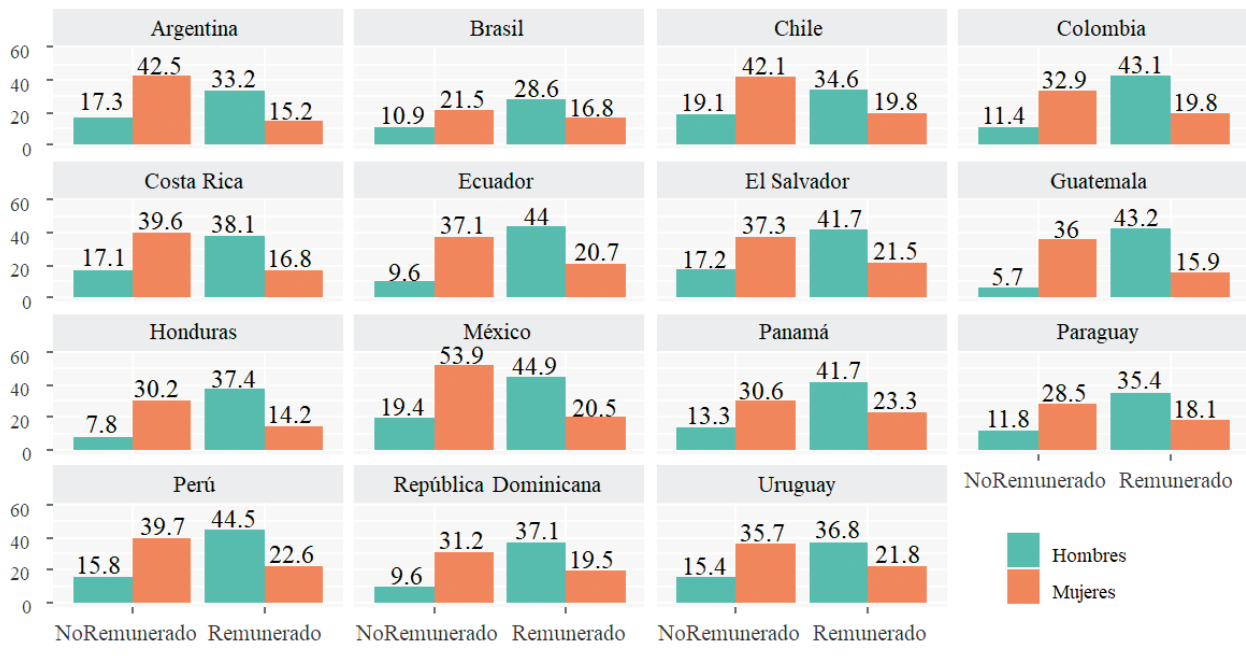

Uruguay

NoRemunerado Remunerado

Figura 4. Tiempo de trabajo remunerado y no remunerado por géneros.

Fuente: elaboración propia en software estadístico R con base en datos Cepal (2020).

En la Figura 5 se presenta la distribución de la población ocupada en cada país según nivel de productividad (baja, media y alta) y género (indicadores POPB, POPM y POPA). Puede notarse que en todos los países latinoamericanos más de la mitad de la población se ubica en sectores de productividad baja y siempre es mayor el porcentaje de mujeres que de hombres con empleos en este sector. En promedio, para todos los países estudiados se considera que el $78.5 \%$ de las mujeres latinoamericanas tienen productividad baja, mientras que tal porcentaje es del $57.9 \%$ entre los hombres.

En contraste, para los sectores de productividad alta se perciben menores diferencias entre géneros, a pesar de que solo un $8 \%$ de las y los latinoamericanos se ubican laboralmente en sectores de productividad alta. Los países en los cuales tal porcentaje supera el $10 \%$, tanto para hombres como para mujeres, son Brasil, Colombia, Costa Rica, Panamá y Uruguay. 

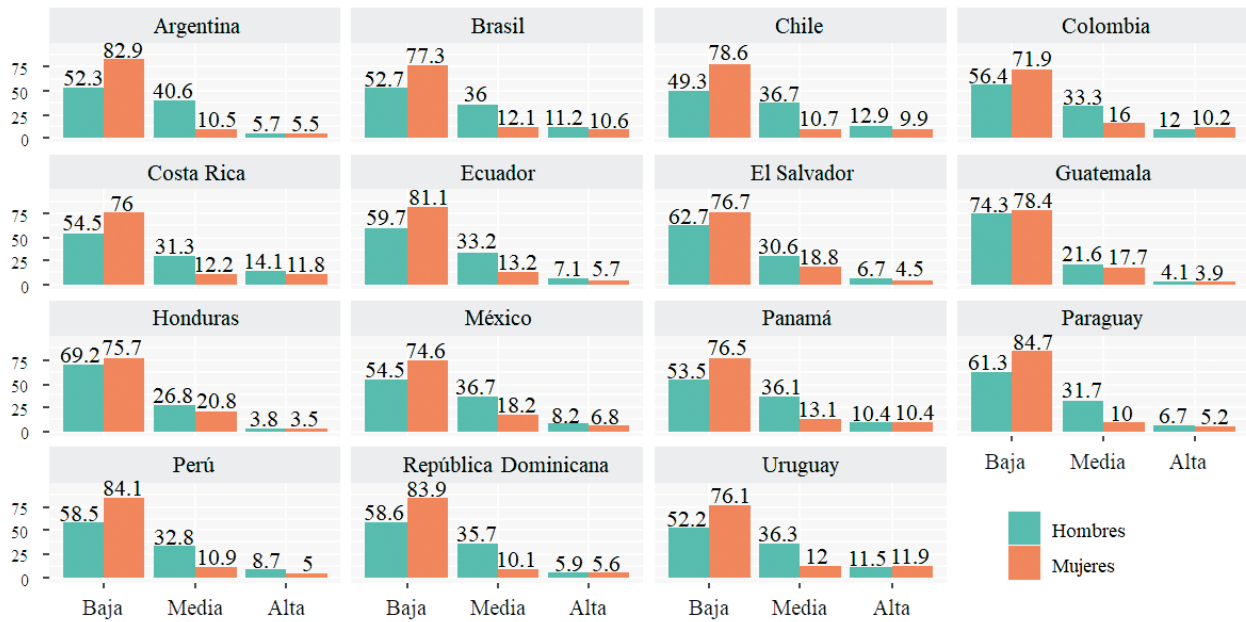

Figura 5. Distribución de la población ocupada según nivel de productividad y género.

Fuente: elaboración propia en software estadístico R con base en datos Cepal (2020).

Antes de examinar la información proporcionada por los resultados del análisis multivariante es pertinente presentar las asociaciones existentes entre los indicadores estudiados, haciendo su distinción entre géneros, según como se presentan en las matrices de correlación en la Figura 6. Se destacan visualmente si son estadísticamente significativas en un nivel de significación $\alpha=0.05$, y se representan en azul las correlaciones positivas y en rojo las negativas. Es posible observar en esta figura que algunos de los indicadores muestran altas correlaciones entre sí, lo cual refleja asociación.
Para el caso de las mujeres (gráfico a la izquierda de la Figura 6), destaca la positiva y alta correlación entre los indicadores PSIP y POPM, con un valor de 0.74 . Esto significa que en la medida en que un país latinoamericano tiene alto el indicador de la proporción de mujeres sin ingresos propios, también presenta una alta participación de estas en sectores de productividad media. En contraste, es baja su participación en los sectores de productividad alta, dado que entre PSIP y POPA la correlación observada es negativa, con valor de -0.48 . 


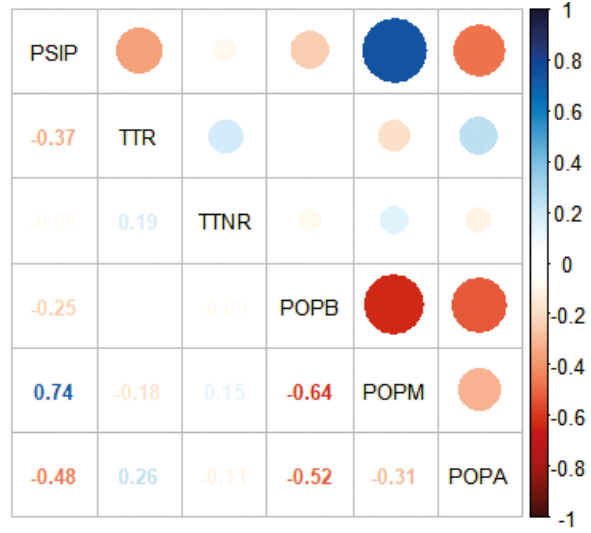

(a) Mujeres

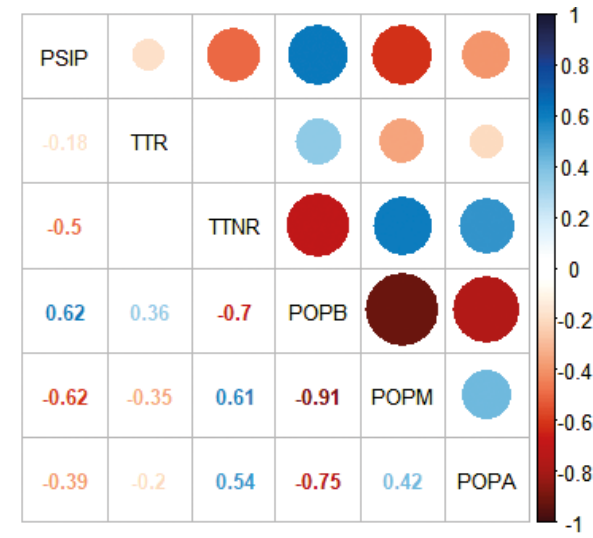

(b) Hombres

Figura 6. Matrices de correlación entre indicadores analizados por género.

Fuente: elaboración propia en software estadístico R con base en datos Cepal (2020).

Ahora bien, respecto a los hombres (matriz a la derecha de la Figura 6), llama la atención la relación inversa entre TTNR, POPB, con un valor de -0.7. Esta asociación muestra que cuando los hombres latinoamericanos se ubican laboralmente en un sector de productividad baja, no declaran dedicar mucho tiempo a las tareas no remuneradas y de cuidado del hogar. Por el contrario, cuando se ubican en sectores de productividad media o alta, sí tienden a declarar tiempo invertido en actividades no remuneradas. Esto último se advierte teniendo en cuenta las correlaciones positivas entre TTNR y POPM, y TTNR con POPA, las cuales son 0.61 y 0.54 , respectivamente (y también son significativas).

En cuanto a los resultados del análisis HJ-Biplot, en la Figura 7 se presenta el primer plano factorial que se obtiene de esta técnica multivariante. Los ejes 1 y 2 recogen el $87 \%$ de toda la variabilidad que existe en relación con las interdependencias de los indicadores de autonomía económica por países, cuando se analiza toda la información, es decir, cuando se consideran los indicadores de cada nación tanto para hombres como para mujeres. 


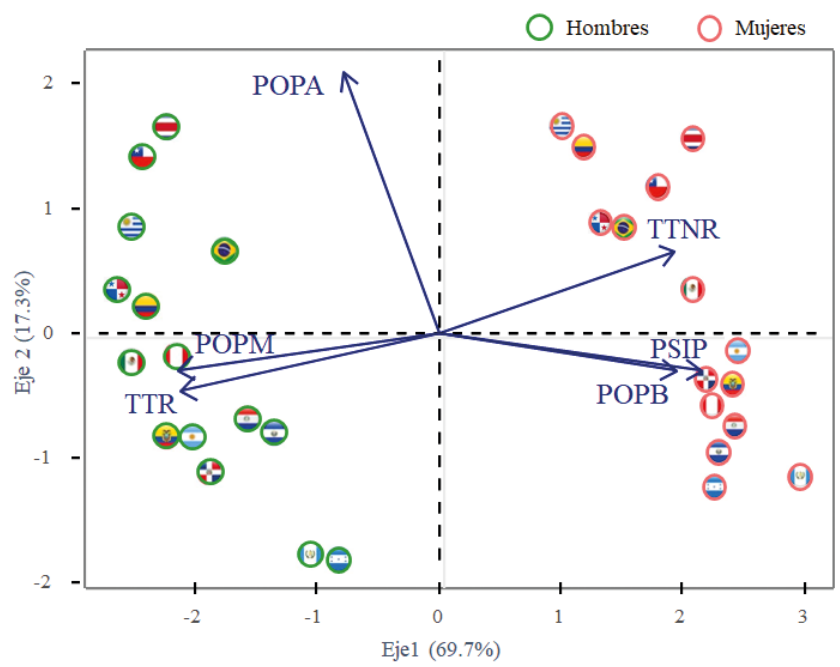

Figura 7. Plano 1-2 del HJ-Biplot de indicadores de autonomía económica.

Fuente: elaboración propia con el programa MULTBIPLOT desarrollado por Vicente-Villardón (2015).

Las direcciones de los vectores que representan a cada indicador marcan una clara diferenciación entre géneros; aquellos que caracterizan a las mujeres se ubican en la dirección del eje 1 (hacia la derecha del gráfico) y, en posición contraria, los que mejor describen a los hombres. Teniendo en cuenta este resultado, las mujeres latinoamericanas están caracterizadas en cuanto a sus condiciones laborales por el tiempo de trabajo no remunerado (indicador TTNR) o por ubicarse laboralmente en sectores de productividad baja (POPB), e incluso, por no tener ingresos propios (PSIP). Sin embargo, los hombres se caracterizan porque declaran mayor dedicación de tiempo al trabajo remunerado (TTR) y pertenecen a sectores de productividad media (POPM).
La diferenciación del vector POPA, que no se observa en la misma dirección de otros vectores, refleja que este caracteriza en conjunto a hombres y mujeres. El hecho de que se ubique en la dirección del eje 2, permite diferenciar la posición de los países que se encuentran en los cuadrantes I y II del plano, de los que se ubican en la parte inferior del gráfico. En la parte superior se encuentran los países que tienen valores más altos en indicadores del porcentaje de su población ocupada en sectores de productividad alta. Es decir, en Uruguay, Colombia, Costa Rica, Chile, Panamá y Brasil se tienen los mayores indicadores de mujeres y de hombres que trabajan en sectores de productividad alta ${ }^{4}$.

$4 \quad$ Ello es posible corroborarlo en las cifras de la Figura 5. 
Dado que al graficar los indicadores de mujeres y hombres en el mismo plano HJ-Biplot (en los ejes 1 y 2), se observan diferencias en la ordenación de los países que se ubican en los cuadrantes III y IV de la Figura 5, se hace necesario un análisis más detallado del comportamiento de los datos, que desagregue la información por géneros, esto es, estudiar por separado a hombres y mujeres. Los primeros planos principales de estos dos análisis HJ-Biplot para cada género se presentan en la Figura 8, donde se pretende identificar de forma más clara las diferencias o similitudes entre naciones según género.

En la Figura 8, la principal diferencia que se observa entre ambas representaciones se asocia a la estructura de covariación ${ }^{5}$ de los vectores correspondientes a los niveles de productividad. En el caso de las mujeres (gráfico a la derecha), los tres niveles (bajo, medio y alto) aparecen distribuidos en el plano en tres direcciones distintas, con ángulos similarmente distanciados entre sí, mientras que en el caso de los hombres la productividad alta y media se ubican en la misma dirección, lo que indica una relación directa entre ambos indicadores. En cuanto a los países, y específicamente en el caso de las mujeres, se observa que Paraguay, República Dominicana, Argentina y Perú forman un conglomerado asociado a sectores de baja productividad (ubicados en el cuadrante III del plano, cerca al vector POPB). También se distingue que Honduras y Guatemala presentan prevalencia del vector PSIP (en la dirección del primer eje, a la derecha del gráfico), que representa a las mujeres sin ingresos propios. Esta última característica no se observaba claramente definida en la representación gráfica del $\mathrm{HJ}$ Biplot de la Figura 7.

En lo que respecta a los hombres (representación izquierda en la Figura 8), Honduras y Guatemala se sitúan en la dirección del vector POPB, lo que demuestra que estas son las naciones con los mayores porcentajes de hombres trabajando en sectores de productividad baja. Brasil, por su parte, tiene un comportamiento independiente y alejado de otros países, en dirección opuesta al vector de tiempo de trabajo remunerado (TTR); es la nación donde los hombres latinoamericanos declararon dedicar menos tiempo a las actividades laborales remuneradas. \begin{tabular}{l}
\hline $\begin{array}{l}\text { Referente a la forma en que se ordenan los vectores en el plano, sus direcciones y los ángulos que se pueden interpretar entre ellos } \\
\text { y en relación con los ejes de reducción de la dimensionalidad. }\end{array}$
\end{tabular} 

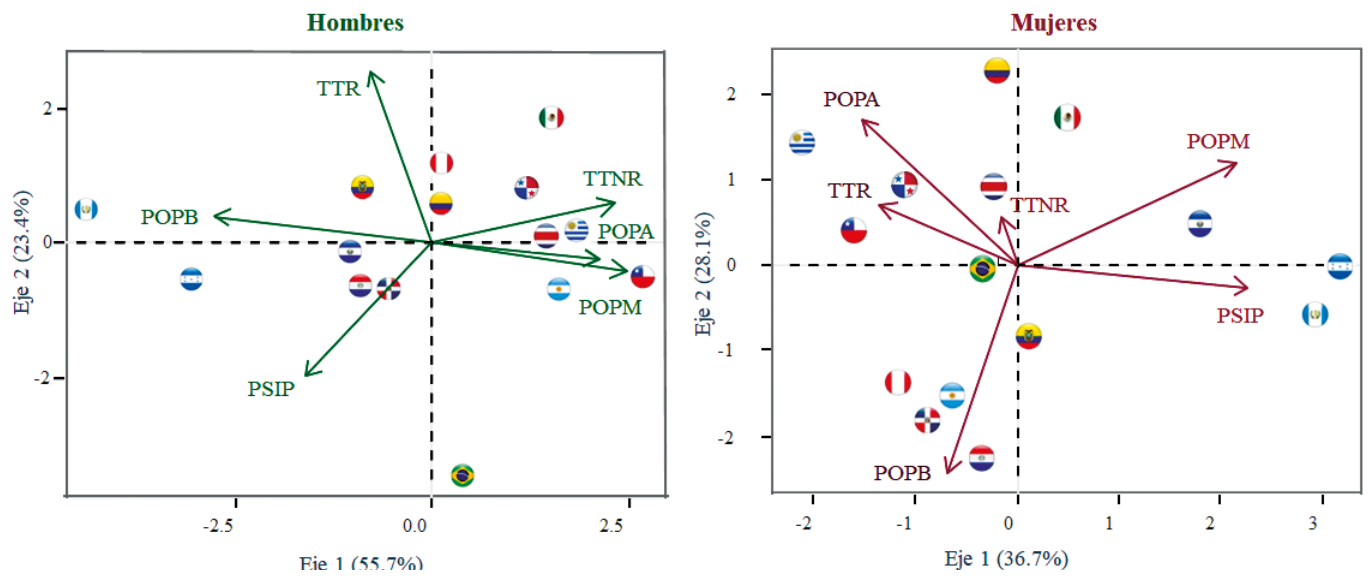

Figura 8. HJ-Biplot (planos 1-2) de indicadores de autonomía económica por géneros.

Fuente: elaboración propia en el programa MULTBIPLOT desarrollado por Vicente-Villardón (2015).

\section{DISCUSIÓN DE RESULTADOS}

Los resultados de esta investigación están en consonancia con la idea de Sabillón et al. (2018), según la cual "la precariedad tiene mayor impacto en las mujeres, una de las razones puede ser su doble presencia y conciliación entre trabajo-familia, así como, diferentes desigualdades que pueden sufrir en sus puestos de trabajo o sus condiciones de empleo" (p. 126). A partir de los resultados obtenidos en este trabajo, ha sido posible establecer que en todos los países latinoamericanos evaluados los porcentajes de mujeres sin ingresos propios son mayores a los de los hombres en condiciones similares. Además, las mujeres tienden a desempeñar trabajos pertenecientes a sectores de productividad baja, lo que concuerda con lo establecido por Basabe-Serrano (2017), en el sentido de que "el mercado laboral en América Latina tiene un claro sesgo que perjudica a las mujeres en términos de enrolamiento" (p. 232).

Países como Honduras y Guatemala fueron los que mostraron mayores valores en los indicadores de dependencia de las mujeres, por no tener ingresos propios. Frente a estos países, Díaz (2019), analizando la situación de pobreza en Centroamérica, explica que estos países tienen "insuficientes tasas de crecimiento económico (respecto a la magnitud de pobreza que tienen), alto grado de concentración del ingreso, altas tasas de criminalidad y de expatriación de población" ( $p$. 150); por lo cual, en estas naciones no solo se registra precariedad en términos de la autonomía económica de las mujeres, sino que también los hombres suelen estar inmersos en situaciones de pobreza. 
En relación con los indicadores de tiempo analizados, el principal resultado obtenido en este trabajo es similar a los hallazgos de muchas investigaciones de género: los datos analizados reflejan inequidad en el uso del tiempo de hombres y mujeres. Esto es consecuencia de una mayor dedicación de las mujeres latinoamericanas a actividades no remuneradas de mantenimiento y cuidado en el hogar, de forma que se limita el tiempo que dedican a las actividades de generación de ingresos propios y se restringen sus posibilidades de acción, "ya que las formas en que las personas pasan su tiempo tienen implicaciones significativas en la calidad de vida" (Kizilirmak \& Köse, 2019, p. 61).

En este estudio se ha encontrado que las mujeres mexicanas son las que declaran la mayor dedicación de tiempo a actividades no remuneradas, en relación con los demás países latinoamericanos. Al respecto, López-Guerra y Rojas (2017) sostienen que ello puede deberse a la feminización del empleo femenino en el país, puesto que "su diversificación se hace a costa del incremento del trabajo de las mujeres, que les implica dobles o triples jornadas: la doméstica familiar para cumplir con las responsabilidades del hogar, la asalariada y la social comunitaria" (p. 346).

Frente a los indicadores asociados a los sectores de productividad en que suelen trabajar los y las latinoamerica- nas, además de que las mujeres "rara vez ocupan los puestos más altos en el ámbito político y empresarial, sus ingresos son generalmente más bajos que los de los hombres, incluso por un trabajo idéntico al de éstos, tienden a ser discriminadas en el mercado laboral" (Řezanková \& Křečková, 2017, p. 373), en este estudio fue posible observar que cuando las mujeres logran posicionarse profesionalmente en sectores de productividad alta, suelen tener condiciones similares a las de los hombres. Esto sucede en particular para Uruguay, Colombia, Costa Rica, Chile Panamá y Brasil.

En estos países, y solo en el caso del $7.5 \%$ de las mujeres que trabajan en sectores como minería, electricidad, gas y agua, actividades financieras e inmobiliarias, entre otras, que facultan el acceso a ingresos remunerados altos, puede decirse que su empoderamiento les facilita "adquirir el poder y control sobre sus propias vidas. Implica la sensibilización, el fomento de la confianza en sí mismas, y un mayor acceso y control sobre sus recursos y acciones" (Naciones Unidas, 2016, p. 191).

En el marco de la relación existente entre los resultados de este trabajo y el cumplimiento de los ODS en Latinoamérica, es oportuno indicar que las brechas identificadas entre países deben entenderse como una oportunidad para el establecimiento de acciones de política pública que velen por el fomento de la igualdad para la 
mujer, de forma que el desarrollo de su autonomía económica se entienda como una condición necesaria para el desarrollo de la región. En este sentido, es primordial que se continúe trabajando por el cumplimiento de la meta ODS 5.a, que busca

emprender reformas que otorguen a las mujeres igualdad de derechos a los recursos económicos, así como acceso a la propiedad y al control de la tierra y otros tipos de bienes, los servicios financieros, la herencia y los recursos naturales, de conformidad con las leyes nacionales. (CEPAL, 2019, p. 33)

\section{CONCLUSIONES}

En términos interpretativos, en este trabajo puede concluirse que, en cinco de los seis indicadores analizados se observaron diferencias estadísticamente significativas entre géneros. Solo en el porcentaje de hombres y mujeres que trabajan en sectores de productividad alta, no hubo distinciones considerables. Por tanto, se evidenciaron oportunidades para el empoderamiento económico de las mujeres que trabaja en sectores productivos más desfavorecidos, que se asocian a la generación de ingresos propios y al tiempo dedicado a las actividades no remuneradas del hogar, dado que estas tienden a ubicarse laboralmente en sectores de productividad media y baja. En especial, se observó que los países centroamerica- nos son los que registran las mayores brechas de autonomía entre los y las latinoamericanas.

En el aspecto metodológico es importante poner de manifiesto la utilidad del análisis HJ-Biplot para, desde una perspectiva multivariante, estudiar los indicadores de género, dado que este método permite interpretar las múltiples asociaciones observadas entre las variables (indicadores) y las características que definen a las observaciones estudiadas (países), examinando las estructuras conjuntas de variación y covariación, y no tener que hacerlo solo interpretando los datos de forma uni o bivariante. Por ello, esta técnica resulta apropiada para futuras investigaciones socioeconómicas con enfoque de género.

Como limitante de este trabajo debe mencionarse que los indicadores analizados aún no reflejan las posibles consecuencias que la pandemia generada por la COVID-19 durante los años 2020 y 2021 está teniendo sobre la autonomía económica de las mujeres. Esto abre una vía para plantear futuras líneas de investigación, dado que, como indica la CEPAL (2021),

la fuerte contracción económica está afectando negativamente la ocupación y aumentando la precarización de las condiciones laborales en la región, lo que en el caso de las mujeres representa un retroceso de más de diez 
años en su participación en el mercado laboral (p. 2)

y así poder analizar comparativamente la situación pre y pos-COVID-19.

En la actualidad, ya han comenzado a plantearse distintas medidas para fomentar y garantizar que en los próximos años aumente el empoderamiento de la mujer latinoamericana en el ámbito económico. Entre ellas, por ejemplo, las acciones acordadas en el Compromiso de Santiago, celebrado en el marco de la XIV Conferencia Regional sobre la Mujer de América Latina y el Caribe, donde se contempló:

Impulsar sistemas financieros que contemplen el acceso y uso por parte de las mujeres, en particular las mujeres con menores recursos, de un conjunto diversificado de servicios y productos de ahorro y de crédito, incluidos el microcrédito y los seguros, entre otros, que sean de calidad y asequibles, brindar acompañamiento técnico para el fortalecimiento de los emprendimientos productivos de las mujeres. (ONU Mujeres \& CEPAL, 2020, art. 22)

Otras acciones que se plantean para incorporar a las mujeres y la igualdad de género en la gestión de la respuesta a la crisis generada por la COVID-19, se asocian a economía del cuidado. Según como indica ONU Mujeres (2020), es preciso

impulsar medidas de política que permitan reconocer, reducir y redistribuir la sobrecarga de trabajo no remunerado que se produce al interior de los hogares por cuidados de salud y cuidado de niñas, niños, personas mayores y personas con discapacidad, y que es absorbida mayoritariamente por las mujeres. (p. 3)

\section{AGRADECIMIENTOS}

A los revisores, por su experiencia y tiempo dedicado a la evaluación de este artículo, ya que sus comentarios y sugerencias han contribuido a mejorar su calidad.

\section{CONTRIBUCIÓN DE LOS} AUTORES

Este artículo es resultado del trabajo conjunto de las coautoras.

\section{FINANCIAMIENTO}

Este trabajo no tuvo ningún tipo de financiamiento institucional.

\section{DECLARACIÓN CONFLICTO DE INTERESES}

Las autoras declaran que no existe conflicto de intereses. 


\section{REFERENCIAS}

[1] Altuzarra, A., Gálvez-Gálvez, C., \& González-Flores, A. (2021). Is Gender Inequality a Barrier to Economic Growth? A Panel Data Analysis of Developing Countries. Sustainability, 13(1), 367-388. https://oi.org/10.3390/su13010367

[2] Basabe-Serrano, S. (2017). Las desigualdades en la representación de mujeres en cortes supremas de América Latina. En S. Blanke \& S. Kurtenbach (coord.), Violencia y desigualdad: Memorias del Congreso ADLAF 2016 (pp. 220-234). Fundación Foro Nueva Sociedad.

[3] Bidegain, N. (2017). La Agenda 2030 y la Agenda Regional de Género. Sinergias para la igualdad en América Latina y el Caribe. Serie Asuntos de Género n. 143. CEPAL.

[4] Calcagni, M. \& Cortínez, V. (2016). Territorios, empoderamiento y autonomía económica: diversas trayectorias para avanzar en equidad de género en Chile. Serie Documento de Trabajo n. ${ }^{\circ} 223$, Grupo de Trabajo Inclusión Social y Desarrollo. Rimisp.

[5] CEPAL. (2017). Advancing the Economic Empowerment and Autonomy of Women in the Caribbean Through the 2030 Agenda for Sustainable Development. Series Studies and Perspectives n. ${ }^{\circ}$ 60. CEPAL.

[6] CEPAL. (2019). La Agenda 2030 y los Objetivos de Desarrollo Sostenible. Una oportunidad para América Latina y el Caribe. Naciones Unidas. http://hdl.handle. net/11362/40155

[7] CEPAL. (2020). Observatorio de Igualdad de Género de América Latina y el Caribe. https://oig.cepal.org/es.

[8] CEPAL. (2021). La autonomía económica de las mujeres en la recuperación sostenible y con igualdad. Informe especial Covid-19. CEPAL.

[9] DANE. (2020). Mujeres y hombres: brechas de género en Colombia. Consejería Presidencial para la equidad de la mujer y ONU Mujeres, Bogotá.

[10] De Clercq, D. \& Brieger, S.A. (2021). When Discrimination is Worse, Autonomy is Key: How Women Entrepreneurs Leverage Job Autonomy Resources to Find Work-Life Balance. J Bus Ethics, 1-18. https://doi.org/10.1007/s10551-021-04735-1

[11] Díaz, G. (2019). El club de la pobreza de América Latina. El caso Guatemala. Aposta, Revista de Ciencias Sociales, 83, 138-152. 
[12] Díaz-Faes, A, González-Albo, B, Galindo, MP \& Bordons, M (2013). HJ-Biplot como herramienta de inspección de matrices de datos bibliométricos. Revista Española de Documentación Científica, 36(1). http://dx.doi.org/10.3989/ redc.2013.1.988

[13] Gabriel, K. R. (1971). The Biplot Graphic Display of Matrices with Application to Principal Component Analysis. Biometrika, 58(3), 453-467. https://doi.org/10.1093/ biomet/58.3.453

[14] Galindo, M. P. (1986). Una alternativa de representación simultánea: HJ-Biplot. Qüestiió,10(1), 13-23.

[15] Goyes, I. (2019). Marco jurídico para la autonomía económica de las mujeres en Colombia. Revista Academia \& Derecho, 10(18), 165-206. https://doi. org/10.18041/2215-8944/academia.18.6001

[16] Hagqvist, E., Toivanen, S., \& Vinberg, S. (2019). The Gender Time Gap: Time Use Among Selfemployed Women and Men Compared to Paid Employees in Sweden. Time \& Society, 28(2), 680-696. https://doi.org/10.1177/0961463X16683969

[17] Htun, M., Jensenius, F. \& Nelson-Núñez, J. (2019). Gender-Discriminatory Laws and Women's Economic Agency. Social Politics: International Studies in Gender. State \& Society, 26(2), 193-222. https://doi.org/10.1093/sp/jxy042

[18] Kizilırmak, A., \& Köse, T. (2019). Determinants of Leisure Time Use in Turkey. Gazi Iktisat ve Iş̧letme Dergisi, 5(1), 60-72. https://doi.org/10.30855/ gjeb.2019.5.1.005

[19] Lamelas, N. \& Aguayo, E. (2010). Desigualdad de género en Latinoamérica desde la perspectiva de los indicadores de desarrollo humano. Revista Galega de Economía, 19(2), 1-15.

[20] Liu, C., Esteve, A. \& Treviño, R. (2017). Female-Headed Households and Living Conditions in Latin America. World Development, 90, 311-328. https://doi. org/10.1016/j.worlddev.2016.10.008

[21] López-Guerra, V. \& Rojas, O. L. (2017). Rezagos en el nivel de autonomía de las mujeres rurales mexicanas en la primera década del siglo XXI. Estudios Demográficos y Urbanos, 32(2), 315-354. https://doi.org/10.24201/edu.v32i2.1644

[22] Meyer, L. (2019). Promoción de la autonomía económica de las mujeres: un desafío para la sociedad chilena. Revista Rumbos TS: Un Espacio Crítico para la Reflexión en Ciencias Sociales, (19), 73-94. 
[23] Micha, A. (2019). Usos y administración de la Asignación Universal por Hijo (AUH): entre el "deber ser" y la autonomía económica de las mujeres. Trabajo y Sociedad, 32, 359-386.

[24] Morgan, R., Dhatt, R., Kharel, C., \& Muraya, K. (2020). Un enfoque parcial de la igualdad de género debilita a los ODS: es hora de una acción transversal. Global Health Promotion, 27(3), 205-208. https://doi.org/10.1177/1757975920949745

[25] Naciones Unidas. (2016). Integrating a Gender Perspective into Statistics. UN Department of Economic and Social Affairs, Statistics Division.

[26] ONU Mujeres \& CEPAL. (2020). Compromiso de Santiago. En XIV Conferencia Regional sobre la Mujer de América Latina y el Caribe, celebrada en Santiago, 27 a 31 de enero de 2020. Naciones Unidas. https://doi.org/10.22201/ iisue.24486167e.2021.173.60669

[27] ONU Mujeres (2020). Covid-19 en América Latina y el Caribe: cómo incorporar a las mujeres y la igualdad de género en la gestión de la respuesta a la crisis. Naciones Unidas.

[28] Řezanková, H., \& Křečková, Z. (2017). Selected Gender Gap Indicators. Comparison of V4 Countries within EU Context. In 20th International Scientific Conference AMSE Applications of Mathematics and Statistics in Economics (pp. 373-386). https://doi.org/10.15611/amse.2017.20.31

[29] Sabillón, J., Aragón, A., \& López, I. (2018). Medición multidimensional de la precariedad laboral en Centroamérica. Revista de Ciencias Sociales, (162), 117-129.

[30] Sehnbruch, K., González, P., Apablaza, M., Méndez, R. \& Arriagada, V. (2020). The Quality of Employment (QoE) in Nine Latin American Countries: A Multidimensional Perspective. World Development, 127, 1-20. https://doi. org/10.1016/j.worlddev.2019.104738

[31] Vaca-Trigo, I. (2019). Oportunidades y desafíos para la autonomía de las mujeres en el futuro escenario del trabajo. Serie Asuntos de Género n. ${ }^{\circ}$ 54. CEPAL. https:// www.cepal.org/es/publicaciones/44408-oportunidades-desafios-la-autonomia-mujeres-futuro-escenario-trabajo

[32] Varea, S. (2020). Género y objetivos de desarrollo sostenible ¿Hacia un futuro feminista? Revista Internacional de Comunicación y Desarrollo (RICD), 3(12), 118-127. https://doi.org/10.15304/ricd.3.12.7020

[33] Vicente-Villardón, J. L. (2015). MultBiplot: A Package for Multivariate Analysis Using Biplots. Departamento de Estadística. Universidad de Salamanca. http:// biplot.usal.es/multbiplot/ 